\title{
Self-induced steps in a small Josephson junction strongly coupled to a multimode resonator
}

\author{
Larsen, A.; Jensen, H. Dalsgaard; Mygind, Jesper
}

Published in:

Physical Review B

Link to article, DOI:

10.1103/PhysRevB.43.10179

Publication date:

1991

Document Version

Publisher's PDF, also known as Version of record

Link back to DTU Orbit

Citation (APA):

Larsen, A., Jensen, H. D., \& Mygind, J. (1991). Self-induced steps in a small Josephson junction strongly coupled to a multimode resonator. Physical Review B, 43(13), 10179-10190.

https://doi.org/10.1103/PhysRevB.43.10179

\section{General rights}

Copyright and moral rights for the publications made accessible in the public portal are retained by the authors and/or other copyright owners and it is a condition of accessing publications that users recognise and abide by the legal requirements associated with these rights.

- Users may download and print one copy of any publication from the public portal for the purpose of private study or research.

- You may not further distribute the material or use it for any profit-making activity or commercial gain

- You may freely distribute the URL identifying the publication in the public portal

If you believe that this document breaches copyright please contact us providing details, and we will remove access to the work immediately and investigate your claim. 


\title{
Self-induced steps in a small Josephson junction strongly coupled to a multimode resonator
}

\author{
A. Larsen* \\ Physikalisch-Technische Bundesanstalt, D-3300 Braunschweig, Federal Republic of Germany \\ H. Dalsgaard Jensen \\ Danish Institute of Fundamental Metrology, DK-2800 Lyngby, Denmark \\ J. Mygind \\ Physics Laboratory I, B 309, Technical University of Denmark, DK-2800 Lyngby, Denmark
}

(Received 10 September 1990; revised manuscript received 27 November 1990)

\begin{abstract}
An equally spaced series of very large and nearly constant-voltage self-induced singularities has been observed in the dc $I-V$ characteristics of a small Josephson tunnel junction strongly coupled to a resonant section of a superconducting transmission line. The system allows extremely high values of the coupling parameter. The current steps are due to subharmonic parametric excitation of the fundamental mode of the resonator loaded by the junction admittance. Using an applied magnetic field to vary the coupling parameter, we traced out half-integer steps as well as the mode steps known from more weakly coupled systems. Based on a singleresonator model, we explain the exceptional size of the steps by the large content of higher harmonics of the Josephson oscillation sustained by the multiple modes of the resonator. The parameters of the junction and the loaded resonator-measured in situ by using the junction as detector-form a consistent set. The dominant system loss originates in the junction but cannot be ascribed to a relevant conductance.
\end{abstract}

\section{INTRODUCTION}

The Josephson junction is a highly nonlinear element and the interaction with its embedding circuitry have been studied extensively over more than two decades; see Ref. 1, and references cited therein.

High-quality Josephson tunnel junctions consisting of two superconducting films separated by a few-nanometerthick tunneling barrier are now routinely fabricated with use of modern thin-film technology. An understanding of the nature of a close coupling to such elements inherently shunted by the self-capacitance of the tunneling structure is imperative for their use in fast electronics. In small tunnel junctions (i.e., with spatial dimensions shorter than $\lambda_{J}$, the Josephson penetration depth), narrow-band coupling can be achieved by resonating out the capacitance by an external low-loss inductive load or, at the socalled Josephson plasma frequency, by the inductance of the tunneling pair current. The internal electromagnetic (Fiske) resonances allow a magnetically tuneable narrowband coupling. In junctions with dimensions larger than $\lambda_{J}$, other internal (soliton or fluxon) resonances may also be used.

In the present paper we report a study of self-induced steps observed in the dc $I-V$ characteristics of a small tunnel junction strongly coupled to a relatively long section of a low-loss superconducting transmission line. Due to its low impedance compared with free space, the line is considered to be electrically open ended along the pe- riphery. Loaded by the admittance of the junction, it constitutes a multimode (nearly full-wave) resonant system which allows us to achieve an exceptionally close coupling. For a given resonance frequency, the coupling parameter, which also depends on the critical current of the junction and the total system loss, can be varied by applying an external magnetic field to the junction, or by changing the system temperature.

With strong coupling we observe a series of large self-induced steps (PMS, parametrically generated mode steps) excited by parametric subharmonic pumping of the fundamental resonant mode. Within narrow parameter ranges, half-integer PMS are also stable. Self-induced mode steps (MS), known from Josephson junctions interacting with resonant transmission line modes in the weak-coupling limit, ${ }^{2-5}$ are also observed. Our measurements show that, in the multimode system with stronger coupling and large harmonic overlap between the mode resonances, the MS seem to become unstable and be dominated by the PMS. Parametrically generated steps in a single-resonance system have been observed in a resistively shunted superconducting quantum interference device (SQUID). ${ }^{6}$ Steps of this type are called harmonic steps in Ref. 1 .

There are several possible applications of the strongly coupled junction-resonator system. Most obvious is to use the system as a narrow-band self-oscillating dc-biased microwave source. The available power is, as for all Josephson oscillators, approximately given by the prod- 
uct of the dc voltage and current at the bias point on the step. The generator impedance, however, is that of the resonator. What appears particularly attractive is that more of such systems can be coupled together via nextneighbor interactions in mutually phase-locked arrays. ${ }^{7}$ The usable power of a phase-locked array oscillator is proportional to the square of the number of systems, while the linewidth is inversely proportional to this number.

Previously, mutual phase locking has been obtained in arrays of small tunnel junctions using an external impedance (eventually a common resonator) in parallel with the whole array (see Ref. 8, and references cited therein). A general technique for analyzing such arrays has been developed by Hadley, Beasley, and Wiesenfeld. ${ }^{9}$ Coupled arrays are advantageous also as microwave detectors, mixers, and amplifiers due to their narrow linewidth and high saturation power.

A straightforward application of the junction-resonator system is to determine in situ the high-frequency propagation constants of thin-film transmission lines by a relatively simple dc measurement of the position and size of the MS and/or PMS in small test samples placed on the wafer. ${ }^{10}$

Finally, the system is ideally suited for the basic study of nonlinear dynamics. Unlike a bare junction, the system is of third or higher order, and so bifurcations and chaos are likely to occur. ${ }^{11}$ Stability analysis of single as well as arrays of strongly coupled systems is important especially with the future high-current-density submicrometer tunnel junctions where it will be very difficult to avoid interactions with resonances in the embedding network.

The paper is organized as follows. In Sec. II the junction-resonator system is described. From the model, it is shown how the resonance frequencies are determined. The form and the size of the step are discussed as function of the coupling parameter within the single-resonance theory. Geometry and fabrication of samples are included in Sec. III. In Sec. IV the experimental setup and measuring procedure are described, and in Sec. V results obtained in measurements on selected samples are reported. Also the breakdown of the single-resonance theory and various loss mechanisms are discussed here. The paper is concluded in the final section.

\section{THEORY}

\section{A. Positions of mode steps, MS}

In Fig. 1 is shown a schematic diagram of a Josephson tunnel junction integrated in a microstrip resonator. The half-length $\ell$ and the width $w$ of the resonator are defined as shown in the figure. The resonator consists of two superconducting thin-film electrodes separated by an insulating dielectric with thickness $d$. The rectangular junction is situated at the center of the resonator. The high-impedance $\mathrm{dc}$ and $\mathrm{rf}$ connections are designed to interfere weakly with the resonator. (a)

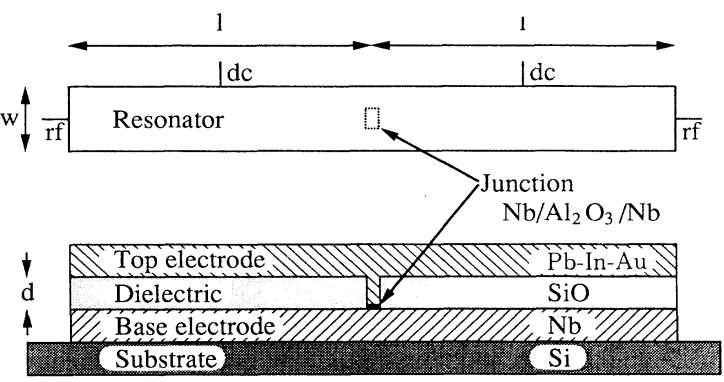

(c)

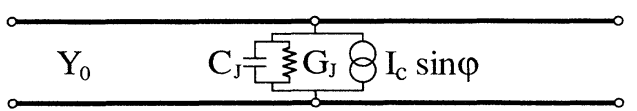

FIG. 1. (a) Top view, (b) side view, and (c) transmissionline model of a microstrip resonator with a small Josephson tunnel junction. The width, half length, and thickness of the dielectric of the microstrip are $w, \ell$, and $d$, respectively. The dimensions are out of scale.

Also shown in the figure is a microwave model of the system. The resonator is an open-ended transmission line with characteristic admittance $Y_{0}$ and propagation constant $\gamma=\alpha+j \beta$. The junction is modeled by a parallel connection of the active element, the supercurrent channel $I_{S}=I_{c} \sin \varphi$, in the following called the bare Josephson element, and the passive admittance $Y_{J}(f)=G_{J}+2 \pi j f C_{J}$, representing the junction capacitance $C_{J}$ and the (in general nonlinear) quasiparticle conductance $G_{J}$. When dc biased at a voltage $V$, the corresponding Josephson oscillation has the fundamental frequency $f=(2 e / h) V$, where $-e$ is the electron charge and $h$ is Planck's constant. For the moment we neglect the admittance, $Y_{S}$, of the bare Josephson element as well as any admittance associated with the change in the current distribution in the films near the junction's periphery. The former assumption is valid for an uncoupled junction operating at frequencies well above its plasma frequency, $f_{p}$. We note that $f_{p}$ in the measurements reported here is 3-5 times larger than the fundamental-mode resonance frequency. However, as discussed in Sec. V, $Y_{S}$ seems to play a minor role.

The admittance $Y_{e}=2 Y_{0} \tanh (\gamma \ell)$ of the two openended microstrip sections transformed to the position of the Josephson element can be calculated with use of ordinary transmission-line theory. The total admittance $Y_{\Sigma}$ seen by the Josephson element then is

$$
Y_{\Sigma}=Y_{J}+Y_{e}=Y_{J}+2 Y_{0} \tanh (\gamma \ell)
$$

and resonance occurs when the imaginary part equals zero; i.e., $\operatorname{Im} Y_{\Sigma}=0$. The corresponding current singularities, which show up in the dc $I-V$ characteristic of the junction even if it is relatively weakly coupled to a resonator, are what we call mode steps (MS) in the following.

Assuming low microstrip losses (i.e., $\alpha \ell \ll 1$ ), $Y_{\Sigma}$ can 
be approximated by

$$
Y_{\Sigma} \simeq G_{J}+G_{e}+j\left[2 \pi f C_{J}+2 Y_{0} \tan \left(\pi \frac{f}{f_{e}}\right)\right],
$$

where $G_{e}=2 Y_{0} \alpha \ell\left[1+\tan ^{2}\left(\pi f / f_{e}\right)\right]$ is the real part of the admittance of the external resonator, and $f_{e}$ is the resonance frequency of the unloaded resonator (without the junction), when one full wavelength of the oscillation equals the length of the resonator. $f_{e}$ is given by

$$
f_{e}=\frac{c_{0}}{2 \ell \sqrt{\epsilon_{r} \chi_{r}}}
$$

where $c_{0}$ is the light velocity in vacuum, $\epsilon_{r}$ the relative dielectric constant of the insulator, and $\chi_{r}=\left(d+\lambda_{a}+\right.$ $\left.\lambda_{b}\right) / d$ accounts for the penetration of magnetic fields in the resonator electrodes. The penetration depths $\lambda_{a}$ and $\lambda_{b}$ should be corrected for the finite thickness of the two electrodes.

As can be derived from Eq. (2), the microstrip sections will act as an inductance for frequencies between $(n-1 / 2) f_{e}$ and $n f_{e}$, where $n$ is an integer, so that resonance occurs only in these intervals. For the resonators examined here, $w$ is on the order of some hundreds of micrometers, while $d$ is about a factor of $10^{3}$ smaller. Hence the effective dielectric constant $\epsilon_{\text {eff }}$ of the transmission line is equal to $\epsilon_{r}$. Likewise, $Y_{0}$ is given by the asymptotic value

$$
Y_{0}=\frac{1}{120 \pi} \sqrt{\frac{\epsilon_{r}}{\chi_{r}}} \frac{w}{d}
$$

With the dimensions used here, $Y_{0}$ is typically a few mhos. At least at the lowest resonance frequencies, the admittance of the junction is much smaller than $Y_{0}$, and in order to fulfill the resonance condition the zero admittances at the open ends of the microstrip therefore has to be transformed to a low admittance at the junction position. This configuration, with low-admittance points at the ends and in the middle of the resonator, corre-

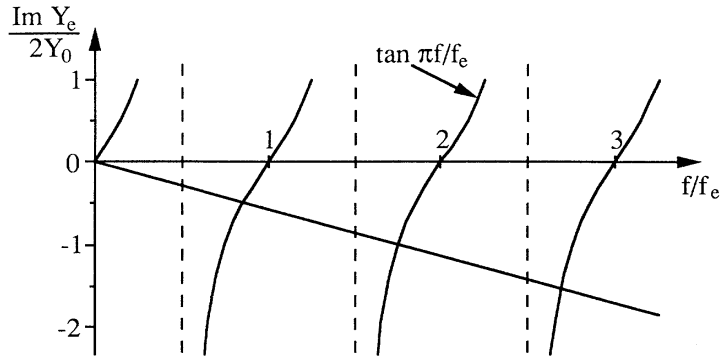

FIG. 2. Graphic solution of Eq. (5). The slope of the straight line is $-\pi C_{J} / C_{e}$. The intersection points determine the mode resonance frequencies $f_{n}$. From the figure it is obvious that $n f_{1}<f_{n}$ for $n=2,3, \ldots$.

sponds to a mode pattern with an integer number $n$ of full wavelengths in the resonator. At higher frequencies, $\left|\operatorname{Im} Y_{e}\right|$ has to be larger than $Y_{0}$ to meet the resonance condition, and so the resonances move towards modes with $\left(n-\frac{1}{2}\right)$ wavelengths.

The resonance condition (low-loss limit) can be written as

$$
\tan \left(\pi \frac{f}{f_{e}}\right)=-\pi \frac{C_{J}}{C_{e}} \frac{f}{f_{e}}
$$

where $C_{e}=\epsilon_{r} \epsilon_{o}(2 \ell w) / d$ is the total capacitance of the microstrip resonator, and $\epsilon_{o}$ is the vacuum permittivity. The solutions to this equation are called $f_{n}$, which are resonance frequencies of the $n$th mode of the system. In the weak coupling limit, the corresponding current singularities in the dc $I-V$ characteristic will be the $n$th mode step (MSn).

In Fig. 2 the graphic solution of Eq. (5) is shown. Resonance occurs at frequencies where the tangent curve intersects the straight line. The slope of this line is $-\pi C_{J} / C_{e}$; thus, a larger $C_{J}$-or for the resonator (with

TABLE I. Mode frequency $f_{n}$, its ratio to the $n$th harmonic of the fundamental-mode frequency $n f_{1}$, attenuation constants $\alpha_{d}$ and $\alpha_{c}$ due to dielectric and surface losses, respectively, real part $G_{e}$ of the transformed resonator admittance $Y_{e}$, theoretical quality factor $Q_{n}^{\text {theor }}$, and coupling parameter $z_{n}$ for the first ten harmonics calculated for parameter values (given in text) typical for sample No. 1. The theoretical quality factor, $Q_{1}^{\text {theor }}$, for the fundamental mode is adjusted to fit $Q_{1}^{\operatorname{expt}}$.

\begin{tabular}{ccccccccc}
\hline \hline$n$ & $f_{n}(\mathrm{GHz})$ & $f_{n} / n f_{1}$ & $\alpha_{d}\left(\mathrm{~m}^{-1}\right)$ & $\alpha_{c}\left(\mathrm{~m}^{-1}\right)$ & $G_{e}\left(\Omega^{-1}\right)$ & $Q_{n}^{\text {expt }}$ & $Q_{n}^{\text {theor }}$ & $z_{n}$ \\
\hline 1 & 8.6 & 1.000 & 0.08 & 0.4 & 0.02 & 101 & 101 & 391.3 \\
2 & 17.2 & 1.003 & 0.16 & 1.3 & 0.06 & 82 & 163 & 139.2 \\
3 & 25.9 & 1.007 & 0.24 & 2.4 & 0.14 & 180 & 194 & 61.2 \\
4 & 34.8 & 1.012 & 0.32 & 3.7 & 0.27 & & 206 & 29.4 \\
5 & 43.6 & 1.017 & 0.41 & 5.2 & 0.48 & & 208 & 15.0 \\
6 & 52.6 & 1.021 & 0.49 & 6.8 & 0.78 & & 205 & 8.2 \\
7 & 61.6 & 1.025 & 0.57 & 8.5 & 1.19 & & 200 & 4.8 \\
8 & 70.7 & 1.029 & 0.66 & 10.1 & 1.75 & & 196 & 2.9 \\
9 & 79.7 & 1.032 & 0.74 & 11.8 & 2.47 & & 192 & 1.9 \\
10 & 88.8 & 1.035 & 0.83 & 13.6 & 3.37 & & 188 & 1.3 \\
\hline \hline
\end{tabular}


given films and dielectric), a larger $d$ or smaller $w$-means that the mode resonance frequencies decrease. Here we have neglected the dependence of $\chi_{r}$ on $d$. This is a good approximation for $d>2\left(\lambda_{a}+\lambda_{b}\right)$. Generally, since $\ell$ enters in $f_{e}$, longer resonators have lower resonance frequencies.

As described above, the first mode resonance frequencies are situated just below $n f_{e}$, while at higher frequencies the resonances lie closer to $\left(n-\frac{1}{2}\right) f_{e}$. It is easily verified, solving Eq. (5), that $f_{n}>n f_{1}$ for $n=2,3$, etc. This is included in Table I, which is calculated with parameter values representative of typical experimental samples, here sample No. 1 . The ratio $f_{n} /\left(n f_{1}\right)$ increases with $C_{J}$ and $d$, and decreases with $w$ and $\ell$; the harmonics of the first (fundamental) mode frequency are closer to the frequencies of the mode resonances for long and wide resonators. We will return to the importance of the harmonic overlap at the end of this section.

\section{B. Single-resonance theory}

Above we discussed the resonance conditions and hence the position of the mode steps, MS, in the multipleresonance-junction-microstrip system. Unfortunately, to our knowledge there exist no theory for the size and shape of the MS.

Self-induced steps may be regarded as a sort of regenerative $r f$-induced steps. At resonance the field in the resonator is large, and the junction receives the resonator signal as driven from a microwave generator, which in turn is powered by the junction itself. This argument was used to explain the form and size of self-induced steps $^{1,12,13}$ in the much simpler system of a small Josephson junction coupled to a circuit with only a single resonance. The important assumption is that harmonics of the Josephson oscillations are negligible when operating near resonance. For use in the following we shall refer to these steps as single-resonator-induced steps (RS, for brevity) and briefly review the main results of this singleresonance theory.

The coupling parameter $z$, which relates the coupling strength to both junction and resonator properties, is defined as

$$
z=\frac{I_{c}}{V_{r} \operatorname{Re} Y_{\Sigma}}
$$

where $I_{c}$ is the critical current of the junction, and $V_{r}=$ $(h / 2 e) f_{r}$ is the dc voltage corresponding to the resonance frequency $f_{r}$.

Figures 12.5 and 12.6 in Ref. $1(m=1)$ show the form and size of the RS as a function of the relative detuning $\xi / z$ and the coupling parameter $z$, respectively. A critical value of coupling exists for $z=2.92$ where the normalized step size reaches its maximum, $I_{s} / I_{c}=0.58$. When $z$ exceeds certain threshold values $(38,130, \ldots)$ the $\mathrm{RS}$ exists in separate current intervals. On rf-induced steps one can also see splitting into current intervals with stable and unstable regions depending on junction parameters, and frequency and amplitude of the applied signal. ${ }^{14,15}$ The splitting of the rf-induced steps is due to bifurcations and chaos. ${ }^{14}$ Numerical simulations on Josephson tunnel junctions coupled to single resonators have also shown bifurcations and chaos. ${ }^{11}$ Therefore the experimentally observed splitting of the RS may indicate chaotic regions.

Also the form of the RS changes as $z$ crosses 2.92. At weaker coupling, the dc voltage across the junction increases monotonically with increasing bias current, looking much like an ordinary resonance curve. With stronger coupling, the RS become more cusplike and may even show a backward bending. The backward bending of steps has been observed in our measurements with the multiple-resonance system. We shall return to this in Sec. V.

For a given step $z$ can be changed by tuning $I_{c}$; in our measurements we apply an external magnetic field. This leaves the denominator in Eq. (6) virtually unchanged. In the undercritical region the height of the RS will decrease rapidly with decreasing $I_{c}$. If, on the other hand, $z$ is larger than $2.92, I_{s} / I_{c}$ will increase with decreasing $I_{c}$, and the decrement in $I_{s}$ will be slower.

The single-resonance theory also predicts parametric subharmonic excitation of the single-resonator system. ${ }^{1}$ When the junction is (current) biased, say, on the quasiparticle curve at a dc voltage equal to $n V_{r}$, the resonator may be parametrically excited. This results in parametrically induced resonator steps (PRS) at these voltages, too. However, the analysis (Figures 12.6, for $m=2$, and 12.10 in Ref. 1) shows that only the second of the PRS is easily excited, i.e., it will appear without external interference. The other PRS are hard to excite, and will appear only if an oscillation with the particular frequency already exists with some minimum amplitude. As mentioned such so-called harmonic steps have already been observed. ${ }^{6}$

\section{Multimode resonator}

After this review we will return to the microstrip junction-resonator system with its multiple resonances. In analogy to the single-resonance theory, the coupling parameter for the $n$th mode may be given as

$$
z_{n}=\frac{I_{c}}{V_{n}\left(G_{J}+G_{e}\right)}
$$

in the low-loss limit. $V_{n}=(h / 2 e) f_{n}$ is the dc voltage corresponding to the mode resonance frequency $f_{n}$.

The quality factor $Q_{n}$ of the $n$th mode can be found from Eq. (2):

$$
Q_{n}=\pi \frac{f_{n}}{f_{e}} \frac{f_{e} C_{J}+G_{e} /(\alpha \ell)}{G_{J}+G_{e}} .
$$

The microstrip losses in $G_{e}$ are all contained in the real part $\alpha$ of the propagation constant, where $\alpha$ is the sum of two terms: surface losses $\alpha_{c}$ and dielectric losses $\alpha_{d}$. For $d$ much smaller than the wavelength of the electromag- 
netic field propagating in the resonator, it is effectively open ended along its periphery and radiation losses are negligible.

The surface loss constant is given by

$$
\alpha_{c}=\frac{R_{a}+R_{b}}{2 w} Y_{0}
$$

where $R_{a}$ and $R_{b}$ are the real parts of the surface impedances of the electrodes. For superconductors the surface impedance can be calculated by using the MattisBardeen theory. ${ }^{16,17}$ Results of these calculations show that $\alpha_{c}$ is strongly frequency dependent (see Table I). Notice that $\alpha_{c}$ is virtually independent of $w$, since $Y_{0}$ in the low-loss model is proportional to the width.

The dielectric loss constant is

$$
\alpha_{d}=\frac{\pi}{2 \ell} \frac{f}{f_{e}} \tan \delta
$$

where $\tan \delta$ is the loss factor of the dielectric. For SiO, it has been measured to be less than $3 \times 10^{-4}$ at $4.2 \mathrm{~K}$ and $9.1 \mathrm{GHz}{ }^{18} \mathrm{As}$ is apparent from Table I, where $\alpha_{d}$ is calculated with use of this value for $\tan \delta$, the dominant losses in the microstrip are due to the surface losses. Changes in $\tan \delta$ with frequency are neglected.

Both $Q_{n}$ and $z_{n}$ have been calculated using representative parameters fitting the measurements in Sec. V. As seen in Table I, except for the first mode, $Q_{n}$ is nearly constant, because at higher modes $G_{e}$ exceeds $G_{J}$, so that $Q_{n} \propto f_{n} / \alpha \ell$ approximately for large $n$. The coupling parameter $z_{n}$ is much larger than 2.92 for the first modes, and rapidly declines with increasing mode number. For high mode numbers, $z_{n} \propto 1 /\left(\alpha \ell f_{n}\right)$.

In other samples $(l, w)=(4.0 \mathrm{~mm}, 250 \mu \mathrm{m})$, the top electrode was symmetrically constricted, leaving a narrow film strip passing over the junction. In this geometry, using a constriction with the same width as the junction, so that the junction is somewhat decoupled from the two halves of the resonator, we observe both integer and half-integer mode resonances in measurements, using the junction as self-detector. Self-induced PMS or MS could not be found at the corresponding voltages. At $4.2 \mathrm{~K}$, we find, for the integer and half-integer mode resonances, $Q_{1}$ $=150$ and $Q_{2}=650$ and $Q_{1 / 2}=640$ and $Q_{3 / 2}=450$, respectively. The half-integer values can be accounted for by the $\alpha_{c}$ values calculated from Mattis-Bardeen theory, indicating that these resonator modes couple very weakly to the junction and, in fact, also that dielectric losses are unimportant at this temperature. Similar calculations of the $Q$ values for the integer mode resonances clearly show that the junction conductance is the dominant system loss. ${ }^{10}$

\section{Harmonic overlap}

In the experiment we have chosen a long and wide resonator. Here, as seen in Table I, the lower harmonics $n f_{1}$ of the fundamental resonance are closer to the $n$th mode frequency $f_{n}$ than the half-power bandwidth of the mode resonances. The resonator, loaded by the junction passive admittance, therefore comprises many high- $Q$ resonant modes being spaced sufficiently constant in frequency to allow for a considerable overlap of harmonics. Consequently, when biased on a given mode resonance, a large number of harmonics of the Josephson oscillation are likely to be excited in the system and the basic assumption of the single-resonance theory is invalidated. The coupling parameter $z_{n}$ thus needs redefinition and may have different critical values.

The existence of finite-amplitude harmonics makes this analysis a complex problem due to the nonlinearity of the junction; and, to our knowledge, no such multiple-resonance theory exists. Recent calculations and measurements ${ }^{19}$ on Josephson junctions with $\mathrm{rf}$ drive at both the fundamental and the second harmonic have shown rf-induced steps larger than the usual Bessel function prediction. So, again regarding the electromagnetic field in the resonator as an rf generator, one could expect larger steps than predicted above for the resonatorinduced steps, especially if the resonator is long and wide. The form and size of both the MS and the PMS in the harmonic overlap case presumably depend strongly on the amplitudes and mutual phase relations of a multitude of harmonics.

Another extrapolation from the single-resonance theory is that, as with the PRS, the size of the higher-order parametrically generated mode steps PMS should also be reduced drastically and disappear for small values of $z_{n}$. From the dependence of $z_{n}$ found in Table I, the fundamental ( $n=1)$ mode is the most favorable to be pumped in the parametric process generating the PMS. Whether the system chooses to excite the mode resonance at which it is actually biased, or to generate a PMS by pumping the fundamental mode resonance-or, in principle, any of the modes available-depends in a complex way on several parameters in the multimode system. However, as discussed below, large harmonic overlap and strong coupling seem to favor the latter occurrence.

\section{SAMPLE GEOMETRY AND FABRICATION}

The samples used in the measurements are based on an all-niobium $\left(\mathrm{Nb} / \mathrm{Al}_{2} \mathrm{O}_{3} / \mathrm{Nb}\right)$ sandwich Josephson junction integrated in the center of a superconducting microstrip resonator. As seen in Fig. 1, the base $\mathrm{Nb}$ layer of the junction is extended to form the bottom electrode of the resonator. The top electrode is formed by a third lead-indium-gold ( $\mathrm{Pb}$-In-Au) alloy film, which is connected to the upper $\mathrm{Nb}$ layer of the junction through a window in the $\mathrm{SiO}$ layer. This window is made smaller than the junction to prevent short circuits between the base layer and the Pb-In-Au film. The SiO layer is the separating dielectric in the microstrip resonator. The bottom electrode of the resonator is $100 \mu \mathrm{m}$ longer and wider than the top electrode.

The dc connections to the junction are formed in the bottom and top electrodes perpendicular to the resonator 
at points marked dc in Fig. 1. These points lie halfway between the junction and the ends of the resonator. When the resonator contains an integer number of wavelengths, the admittance transformed from the open end will be very large at the position of the dc connections. Thus these will not disturb the resonator, as verified by microwave calculations.

The rf connection are $10-\mu \mathrm{m}$-wide lines, extending from the ends of the resonator. The lines are 10-20 times narrower than the resonator, and outside the bottom electrode they are themselves microstrip lines with a thick copper foil positioned $0.5 \mathrm{~mm}$ above the sample as ground plane. Due to the large impedance mismatch, the load on the resonator from the rf connections can be neglected. A tapered microstrip transformer reduces the line impedance to $50 \Omega$ to match the microstrip-coaxial launcher at the edge of the chip. The rf circuit includes a dc block made on the chip.

Eight $25.4 \mathrm{~mm} \times 12.7 \mathrm{~mm}$ samples are made in each batch on a 3 -in. diam. high-resistivity $\mathrm{Si}$ wafer. In a given batch the junctions show less than $5 \%$ variation in $I_{c}$. Only the resonator dimensions differ between the samples on a wafer, using $\ell=2.75,4.0$, and $5.5 \mathrm{~mm}$, and $w=100$ and $200 \mu \mathrm{m}$. The size of the rectangular junction is $20 \mu \mathrm{m} \times 30 \mu \mathrm{m}$.

The fabrication procedure is the following. The $\mathrm{Nb} / \mathrm{Al}_{2} \mathrm{O}_{3} / \mathrm{Nb}$ sandwich is made, covering the whole surface of the wafer. First the $\mathrm{Nb}$ base layer is deposited by sputtering with a rate of about $1 \mathrm{~nm} / \mathrm{s}$ in an argon plasma. The thickness of the film is about $110 \mathrm{~nm}$. The aluminum layer is likewise sputtered in argon with a deposition rate of approximately $10 \mathrm{~nm} / \mathrm{min}$, reaching a film thickness of $20 \mathrm{~nm}$. The aluminum is then oxidized in a flow of pure oxygen (pressure $\sim 500 \mathrm{mb}$ ) at $120^{\circ} \mathrm{C}$ for $1 \mathrm{~h}$. Finally the $110-\mathrm{nm}$-thick top $\mathrm{Nb}$ layer is deposited under the same conditions as the base layer. For current densities about $100 \mathrm{~A} / \mathrm{cm}^{2}$ the specific capacitance is 4.7 $\mu \mathrm{F} / \mathrm{cm}^{2}$.

The junctions are patterned by a photolithographic process, and the $\mathrm{Nb}$ top layer is reactive ion etched in $\mathrm{SF}_{6}$ gas. Without removing the photoresist, the uncovered surface of aluminum oxide and the $\mathrm{Nb}$ base layer are anodized $(20 \mathrm{~V}, 10 \mathrm{~min})$ in an electrolyte $(126 \mathrm{~g} \mathrm{am}-$ monium pentaborate, $1124 \mathrm{ml}$ ethylene glycol, and 1260 $\mathrm{ml}$ distilled water). In a new photolithographic process, the base layer is then patterned and reactive ion etched in $\mathrm{CF}_{4}$ gas.

After yet another photolithographic patterning with a photoresist for liftoff, the $\mathrm{SiO}$ dielectric is thermally evaporated. The rate is $1 \mathrm{~nm} / \mathrm{s}$, and the total thickness $d=$ $300 \mathrm{~nm}$. The evaporation takes place in a flow of pure oxygen. This reduces the chances of pinholes through the dielectric. The anodization ensures that even if pinholes should exist, the nonconductive surface of the base electrode prevents short circuits.

After liftoff in acetone, the pattern for the 550-nmthick $\mathrm{Pb}-\mathrm{In}(9.5$ wt. \%)-Au(4.8 wt. \%) alloy top electrode is made in a fourth photolithographic process. The sur- face of the $\mathrm{Nb}$ top layer of the junction sandwich is cleaned in an argon plasma just before deposition of the top electrode by thermal evaporation. Lead and indium are deposited first at a rate of about $1 \mathrm{~nm} / \mathrm{s}$ and then the gold at about $0.2 \mathrm{~nm} / \mathrm{s}$. The uncertainty of the thickness of the various layers is $\sim 10 \%$.

Some of the samples are also used in our study of mutual phase locking of Josephson tunnel junctions. ${ }^{7}$ On these samples two identical resonators each containing one junction are placed parallel to each other 4 and 8 $\mu \mathrm{m}$ apart. The separation, however, is much larger than $d$, so that the coupling between the resonators is small. This mutual coupling is of no importance for the results reported here.

\section{MEASURING SYSTEM}

The sample is mounted in a small sample holder with a launcher connecting to the coaxial waveguide leading to the room temperature microwave setup. A thick copper foil provides the ground plane in the microstrip circuit leading from the rf connections on the superconducting resonator (see Fig. 1) to the launcher. The sample holder is placed in the center of a magnetic coil with a separate battery-powered current supply. The magnetic field is applied in the plane of the junction parallel to the long side of the resonator. In the measurements reported here, the sample holder is emersed directly in liquid helium contained in a 10-l superinsulated cryostat equipped with a full-length double Cryoperm magnetic shield. The temperature can be stabilized in the range $4.2-1.5 \mathrm{~K}$ by regulating the bath pressure.

The dc current bias can be supplied either from an electronic unit with sweep and various offset facilities, or from a manually operated, purely resistive circuit. The latter is used for the linewidth measurements (see below), where the bias noise is a critical parameter. A specially designed low-noise, minimum-drift instrumentation amplifier detects the dc voltage across the junction. For accurate measurements of the step voltage, or in situations where the voltage amplifier is disconnected, we record the frequency of the emitted Josephson oscillation. Two junctions can be biased independently and measured simultaneously. The absolute calibration of recorded dc $I-V$ characteristics relies on precision resistors, and the actual position of rf steps induced by microwave power applied to the junction from a synthesized sweep generator. The above-mentioned sweep and offset facilities are used also when we record the magnetic-field dependence of the critical current and the size of the various rf- and self-induced steps.

All sensitive electronic parts are powered by batteries and large efforts have been made to reduce interference from external noise sources. The wires leading to the junction are carefully filtered, twisted pairs. The importance of the stability of the three dc-bias parameters (temperature $T$, applied magnetic field $B$, and bias current $I$ ) is illustrated by the measured (typical) frequency 
tuning rates of the Josephson oscillation when biased on one of the PMS: $\Delta f / \Delta T=-0.15 \mathrm{MHz} / \mathrm{mK}, \Delta f / \Delta B=$ $0.5 \mathrm{MHz} / \mu \mathrm{T}$, and $\Delta f / \Delta I=2 \mathrm{MHz} / \mu \mathrm{A}$ (with a dynamic resistance of about $10 \mathrm{~m} \Omega$ ), respectively.

In order to characterize the samples and as a diagnostic tool for eliminating noise sources, we measure the escape rate from the zero-voltage state. Usually we collect 50000 escape events in an automated data-processing system. Typically we find an effective temperature a few tenths of a kelvin above the bath temperature. The escape-rate measurements allow us to correct for noise suppression of the critical current.

Microwave circulators, isolators, and rejection filters inserted in the coaxial cable minimize the influence of unwanted reflections, harmonics, and extraneous interference. The microwave-detection system consists of a series of low-noise field-effect transistor (FET) amplifiers and a high-quality spectrum analyzer. In the band 2-20 $\mathrm{GHz}$, the noise figure is $\sim 4 \mathrm{~dB}$ and the spectral resolution is $10 \mathrm{~Hz}$. The reference oscillators of the spectrum analyzer and the synthesized microwave generator are locked together. This is very convenient especially when we want to precisely map out a step or to phase lock the Josephson oscillation on a given step. The large spectral coverage $(2-26 \mathrm{GHz})$ of the microwave generator allows us in most cases to measure resonance frequency and quality factor of three or more modes of the loaded resonator. The use of the Josephson junction as detector when biased on the gap structure is discussed in the next section.

\section{EXPERIMENTAL RESULTS}

\section{A. dc properties}

Figures 3 and 4 show the low-voltage part of the dc $I-V$ characteristics of two junctions (No. 1 and No. 2) from the same wafer. Similar characteristics have been obtained for all the other junctions on the wafer. The results reported in this paper are typical also for junctions from other wafers, however, to avoid confusion and in order to emphasize the influence from the resonator we have chosen to discuss only results measured on these two samples with nearly identical junctions.

All the junctions on the wafer were measured to have the same parameters, e.g., area $30 \times 20 \mu \mathrm{m}^{2}$ and $I_{c}=775$ $\mu \mathrm{A}$ at $4.2 \mathrm{~K}$. Sample No. 1 has the longest and widest resonator on the wafer, $\ell=5.5 \mathrm{~mm}, w=200 \mu \mathrm{m}$, while No. 2 has the shortest and narrowest one, $\ell=2.75 \mathrm{~mm}$, $w=100 \mu \mathrm{m}$. The critical current is corrected $(\sim 3 \%)$ for noise suppression found from escape-rate measurements as described above.

Both samples showed nearly perfect magnetic interference patterns; the first lope is shown in the upper part of Fig. 5, indicating an undisturbed tunneling barrier with constant current density. From the $I_{c}(B)$ minima and from the position of the Fiske step, at $295 \mu \mathrm{V}$, we derive the magnetic thickness, $d_{J}=225 \mathrm{~nm}$, the capacitance $C_{J}$
$=28 \mathrm{pF}$, and the Josephson penetration depth, $\lambda_{J}=30$ $\mu \mathrm{m}$ at $4.2 \mathrm{~K}$. The normalized length $L / \lambda_{J}=1$ and the maximum plasma frequency $f_{p}=46 \mathrm{GHz}$. Corrected for the finite thickness $(110 \mathrm{~nm})$ of the $\mathrm{Nb}$ films and the $\mathrm{Al}$ layer $(20 \mathrm{~nm})$, the value found for $d_{J}$ is in accord with the bulk value of the London penetration depth in niobium $(86 \mathrm{~nm})$.

These values together with the resonator parameters were used to calculate the entries of Table I. The parameter $\chi_{r}$ in Eq. (3) is calculated with $\lambda_{a}+\lambda_{b}=160 \mathrm{~nm}$; the London penetration depth in the $\mathrm{Pb}-\mathrm{Au}$-In film was determined to $67 \mathrm{~nm}$ at $4.2 \mathrm{~K}$ in an independent determination of the electron mean free path $l$ in four-point resistance measurements performed on separate samples. These samples were fabricated with well-defined geometry by deposition of $\mathrm{Pb}-\mathrm{In}-\mathrm{Au}$ films on a silicon wafer, using the same composition and procedure as described above in Sec. III. At $4.2 \mathrm{~K}$, we calculate $l=45 \mathrm{~nm}$ for the $\mathrm{Pb}-\mathrm{In}(9.5 \mathrm{wt.} \%)-\mathrm{Au}(4.8 \mathrm{wt} . \%)$ alloy top electrode. The small value found for the penetration depth may be ascribed to the stoichiometric composition of the alloy. According to the Mattis-Bardeen theory ${ }^{16} \chi_{r}$ varies less than $5 \%$ with frequency up to $440 \mathrm{GHz}$. The relative dielectric constant, $\epsilon_{r}=5.7$, of the $\mathrm{SiO}$ insulator is gen-
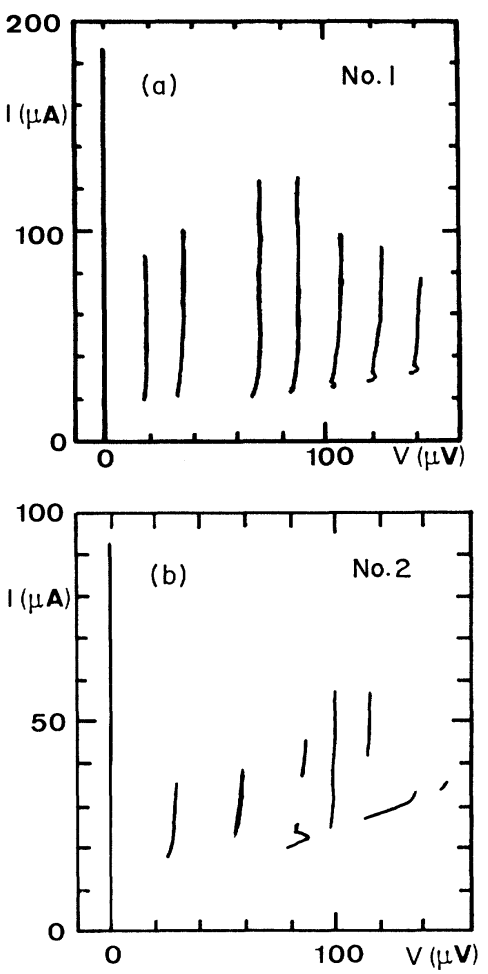

FIG. 3. Self-induced steps measured at $4.2 \mathrm{~K}$ on two samples with identical Josephson tunnel junctions: sample No. 1, $\ell=5.5 \mathrm{~mm}, w=200 \mu \mathrm{m}, B=266 \mu \mathrm{T}$, and sample No. 2, $\ell=2.75 \mathrm{~mm}, w=100 \mu \mathrm{m}, B=295 \mu \mathrm{T}$, respectively. Each picture is a reproduction of multiple exposure oscillographs taken with swept bias current. 


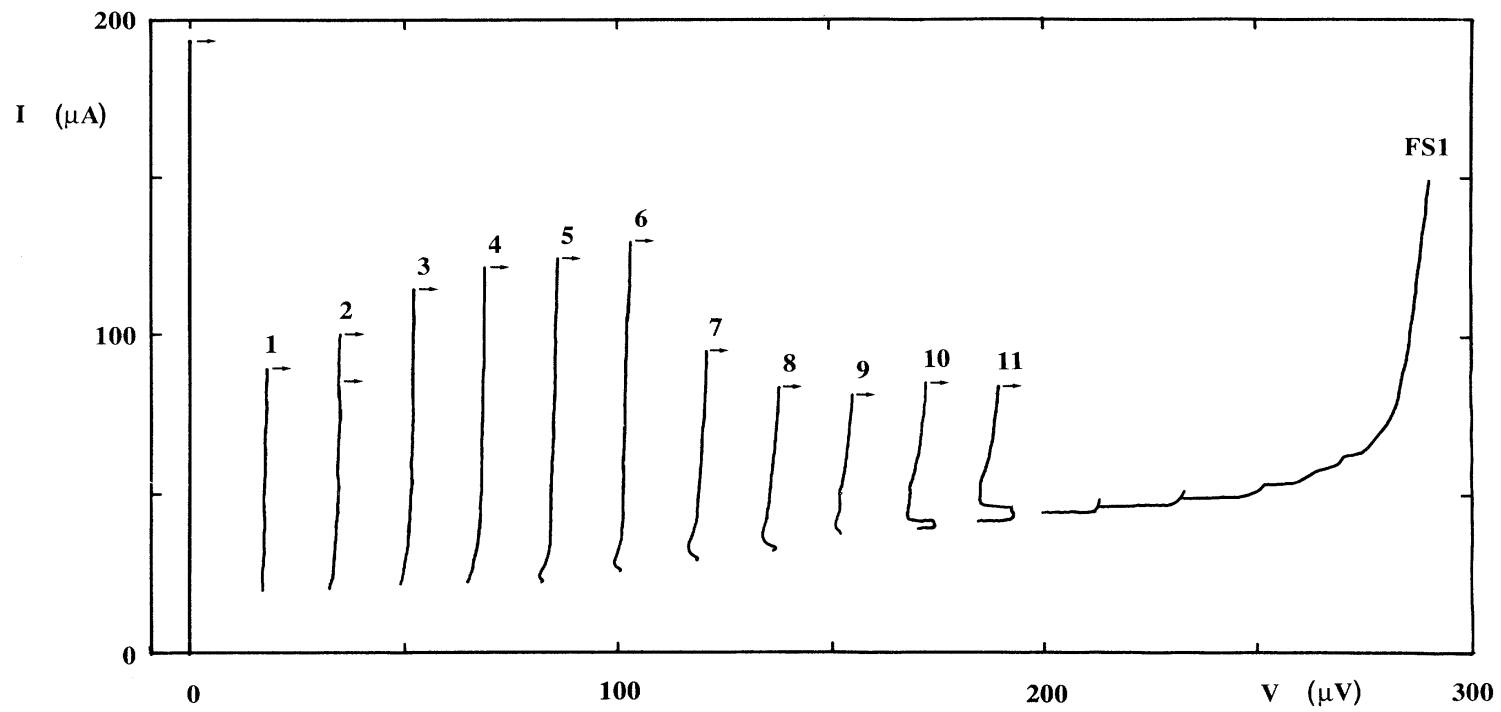

FIG. 4. Recorded dc $I-V$ characteristic of sample No. 1 showing the first mode step, MS1 and a series of parametrically generated mode steps, PMS $k$. The temperature was $4.2 \mathrm{~K}$ and an external magnetic field $B=272 \mu$ T was applied. The arrows indicate the current at which the junction switches from the step. The structure to the right is the first Fiske step at 295 $\mu \mathrm{V}$. The position of the structures visible on higher-numbered PMSk agree with the voltages of the corresponding mode steps, $\mathrm{MS} n$.

erally accepted to be independent of frequency at our experimental frequencies. ${ }^{20}$

In Fig. 3, the magnetic field was chosen to give large steps, and clearly the steps are smaller for the No. 2 junction, both absolute and relative to $I_{c}$. This agrees with the supposition in the last part of Sec. II. Sample No. 1 has the longest and widest resonator of the two, so both the maximum coupling parameter and the contents of harmonics of the resonance oscillation frequency should be larger for this sample. On the dc $I-V$ characteristics of junction No. 1 depicted in Fig. 3 the third step is missing. However, at other magnetic fields this step also appears.

The step structure extends to higher voltages as shown in Fig. 4 in sample No. 1 up to the first Fiske step at $295 \mu \mathrm{V}$. Actually we have in this sample occasionally observed steps also in the region between the first and second Fiske step, as well as a satellite structure with the same periodicity around the first Fiske step.

The microwave radiation emitted from the steps is measured as described in Sec. IV. The linewidth of the detected signal is very narrow, down to a few $\mathrm{kHz}$, so a precise measurement of the Josephson frequency and hence the step voltage is possible. For junction No. 1 the frequency at MS1 is $\sim 8.4 \mathrm{GHz}$ corresponding to a voltage of $17.4 \mu \mathrm{V}$. Radiation at the same frequency has been measured on all stable steps and the variation in frequency from step to step is much smaller than the predicted variation of the mode resonances $f_{n}$ (see Table I). Thus all the steps are harmonic replicas of the fundamental-mode step, MS1 - a strong evidence for our interpretation of the harmonic steps being generated by parametric subharmonic pumping of the fundamental-

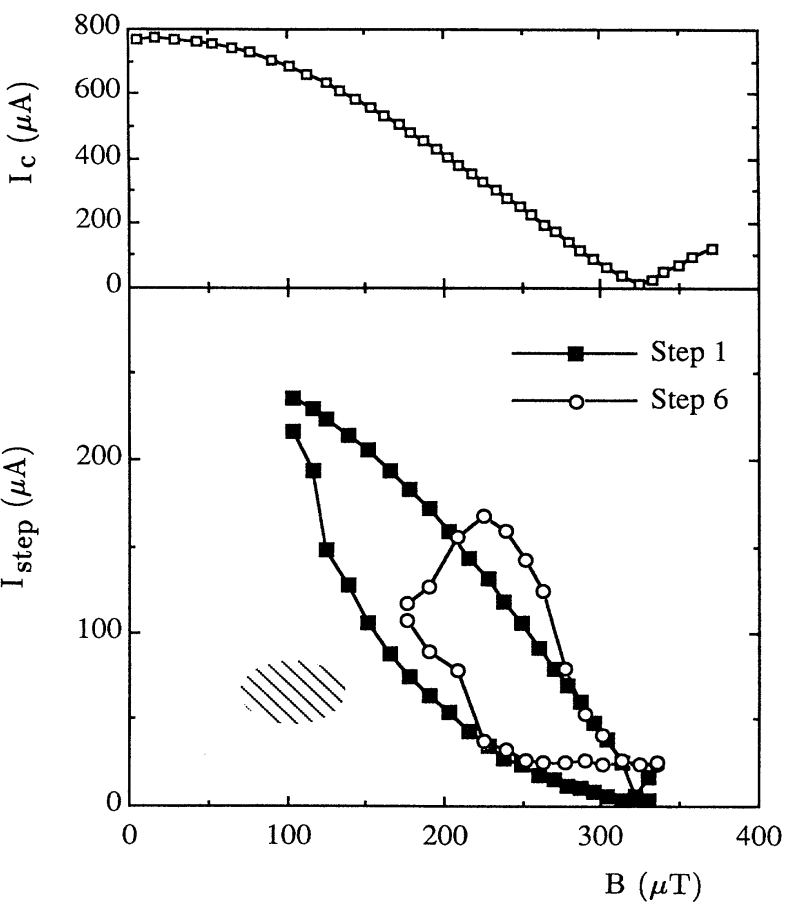

FIG. 5. Critical current $I_{c}$ and stability range of selfinduced steps vs magnetic field for sample No. 1. Step 1 is the fundamental resonance step MS1 at $17.5 \mu \mathrm{V}$, and step 6 the sixth parametrically generated mode step PMS6 at 105 $\mu \mathrm{V}$. Symbols and 0 indicate the upper- and lower-current values at which the junction switches from the step. MS1 has also been observed in the hatched area (see text). The small offset of $+17 \mu \mathrm{T}$ is due to a permanent magnetic field in the cryostat. 
mode step. The $\sim 2 \%$ difference between the measured frequency on MS1 and $f_{1}$ in Table $I$ is easily accounted for by the experimental uncertainties discussed above.

With reference to Sec. II, we use in the following the abbreviation PMS $k$ for these steps. Label $k$ indicates that it is the $k$ th subharmonic of the Josephson oscillation which pumps the fundamental-mode resonance. As shown below the system selects the fundamental mode, which has the largest coupling parameter in our experiments. In principle, one could expect pumping of other modes.

Large signals are also radiated at the second harmonic of the fundamental-mode frequency, i.e., at $16.8 \mathrm{GHz}$, on all steps. This further supports the presence of many large-amplitude higher harmonics, and the invalidity of the single-resonance theory. The structure visible at the bottom of each of the higher-numbered PMS $k$ agrees with the position of the corresponding $(n=k)$ mode steps (MSn), according to Table I.

The frequency of the radiated signal from a step with fixed bias current can be tuned by varying the temperature and the applied magnetic field. At $8.4 \mathrm{GHz}$, we find the same thermal tuning rate $\Delta f / \Delta T \approx-150 \mathrm{kHz} / \mu \mathrm{K}$ on all steps, supporting the interpretation of a subharmonic pumping of the fundamental mode. The magnetic tuning rate at fixed bias current for PMS $k$ increases linearly with increasing $k$, as $\Delta f / \Delta B=0.52+0.13 k \mathrm{MHz} / \mu \mathrm{T}$. We have no explanation for this dependence, but suspect that it is related to the change of bias current relative to the step size. On the PMS $k(k=6,8$, and $9 ; B=272$ $\mu \mathrm{T}$ ) shown in Fig. 4 , we observed $\Delta f / \Delta I \leq 0$ in narrow current ranges. We return to this backward bending below. Also on a few rather irregular PMSk $(k \geq 9)$, we found bias points where $\Delta f / \Delta B=0$.

The MS1 and the PMSk are nearly vertical with the cusplike form predicted in the single-resonance theory for high values of the coupling parameter. In many cases we observed a strange hybridization of the steps, each step appearing split into two, one of which was more stable. The two sets of steps had a difference in resonance frequency of $\sim 150 \mathrm{MHz}$. This splitting, however, has been experimentally traced back to unexpected reflections in the long coaxial cables in the external microwave circuit acting back on the system. This stresses the necessity of avoiding high- $Q$ spurious resonances in the embedding network, especially the low-frequency ones, which inherently might have the largest coupling parameter.

The MS1 of sample No. 2 is at $30.5 \mu \mathrm{V}$ as shown in Fig. 3. In addition to the PMSk, a step at $100 \mu \mathrm{V}$ is observed. Making the same calculations as in Table I, using the resonator dimensions of sample No. 2, we find the fundamental resonance step, MS1, at $30.3 \mu \mathrm{V}$ and the third mode resonance step, MS3, at $100.0 \mu \mathrm{V}$; MS3 is larger than MS1 indicating that the coupling parameter $z_{3}$ is closer to the critical value at the third step, and since $z_{n}$ decreases with the mode number (Table I), $z_{1}$ must be overcritical for the fundamental step.

As mentioned above, the calculated mode resonance frequencies $f_{n}$ are in good agreement with the measurements. This indicates, as assumed in Sec. II, that the admittance of the bare Josephson element does not significantly perturb the determination of the resonance frequencies. We shall return to this below. The imaginary admittances which could be anticipated due to the concentration of the current paths near the periphery of the junction also seem to be unimportant. Interference from the internal junction resonances (e.g., Fiske steps, soliton resonances) appears to be negligible.

In Fig. 3 the largest step for junction No. 1 is the PMS5. At larger magnetic fields (see Fig. 4), the largest step is a higher-numbered step. Figure 5 shows the dependence on external magnetic field of MS1 and PMS6 within the first lobe of the magnetic interference pattern, $I_{c}(B)$. Both upper and lower bounds of the steps are indicated. Due to a small permanent magnetic field in the cryostat, the maximum of the supercurrent is shifted by $17 \mu \mathrm{T}$. With increasing field, the size of PMS6 increases to a maximum and then decreases again. The other PMS $k$ exhibit similar behavior. The hatched area in Fig. 5 indicates a region where MS1 has been seen on the oscilloscope. The step was not sufficiently stable to be traced out on the slower $X-Y$ recorder. Nevertheless, the appearance of the step in two separate intervals of bias current is another indication of an overcritical coupling according to Fig. 12.6 in Ref. 1.

Since the single-resonance theory does not apply to our experimental system with large-amplitude harmonics of the Josephson oscillation, a direct determination of $z_{n}$ is not possible from the measured step form or step size. In the following, we devise an alternative method to estimate the coupling parameter from measurements of the system losses using the junction as detector.

\section{B. Mode-coupling parameters and system losses}

A small Josephson junction can be used as a very sensitive broadband microwave detector when biased at a fixed dc current on the quasiparticle curve in the vicinity of the superconducting gap. In the small-signal limit, the variation of the dc voltage across the junction is proportional to the power of the applied microwave signal. Figure 6 shows the voltage variation as a function of frequency near the fundamental-mode resonance for junction No. 1. A constant amplitude microwave signal was supplied to the sample via the rf connection shown in Fig. 1. The quadratic power dependence has been carefully checked and the measurements have been carried out at different bias currents. Only the sensitivity changes with the bias point.

In the bias point (about $2.7 \mathrm{mV}$ ), the frequency of the Josephson oscillations is much larger than any system resonance frequency as well as the frequency of the applied microwave signal. Hence the admittance of the bare Josephson element can be neglected and the junction in situ measures the admittance of the resonator loaded by the passive admittance of the junction, $Y_{\Sigma}$ in Eq. (2). 


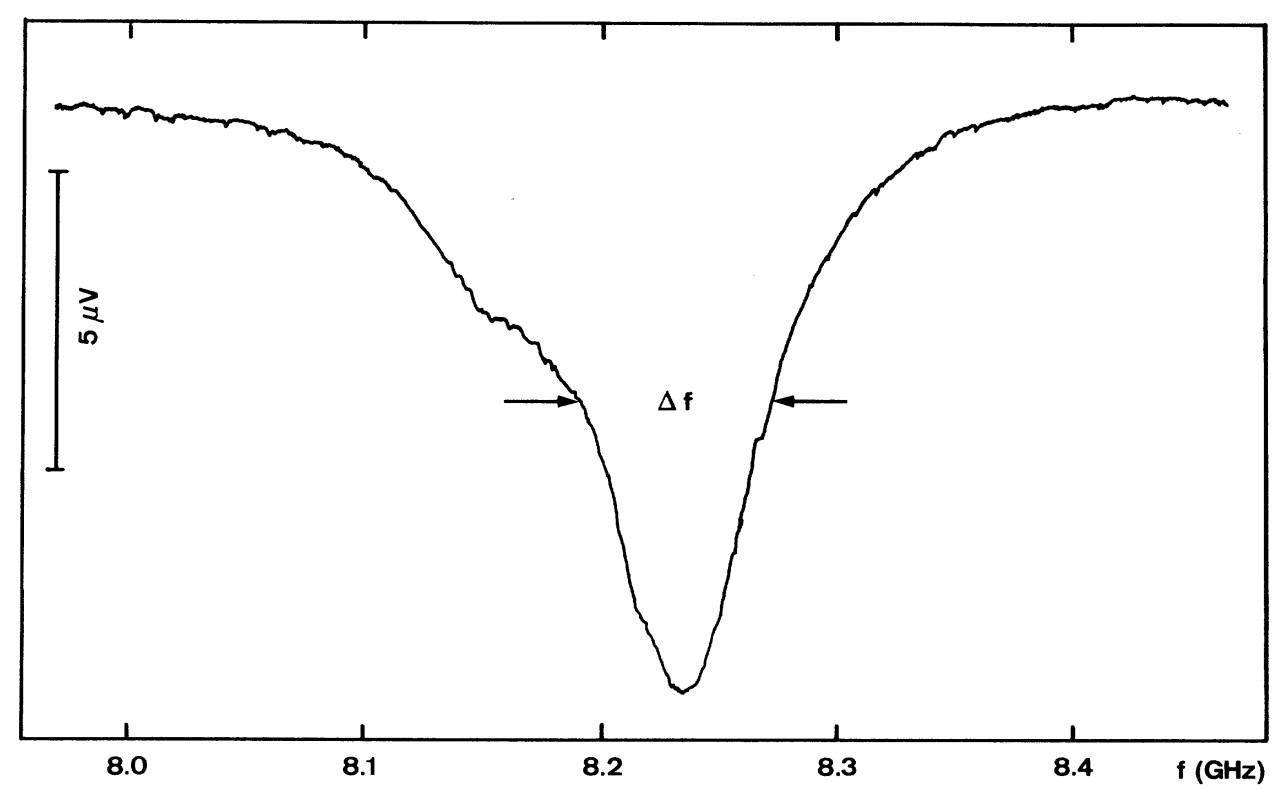

FIG. 6. The dc voltage response $\Delta V$ of the junction vs frequency of an applied microwave signal near the fundamental-mode resonance frequency $f_{1}$. The junction was used as a square-law detector biased just below the gap voltage, and a small swept microwave signal of constant power was supplied via the rf connections to the resonator. The quality factor $Q_{1}$ is evaluated from the frequency width $\Delta f$ at the half-power points.

This is strongly supported by the experimental fact that the curve shown in Fig. 6 is independent of the applied magnetic field. The major source of error is the assumption of a constant power supplied by the high-impedance rf connections at the end of the sample. In fact, the detector response is folded with the frequency-dependent transmission coefficient of the cables, isolators, etc., and a weak interference pattern superimposed on the resonance dip is visible in Fig. 6. The frequency at the maximum drop in voltage is $f_{1}=8.236 \mathrm{GHz}$.

Biased at the top of MS1 with an applied magnetic field $B=272 \mu \mathrm{T}$, we observe Josephson radiation at 8.364 $\mathrm{GHz}$. The discrepancy with the $8.236 \mathrm{GHz}$ value cannot be explained by magnetic tuning, and we propose two explanations: (i) The highest frequency detected may come from a bias point being slightly below the top of the step. As the step is backward bending, similar to what we find in the single-resonance theory, ${ }^{1}$ external noise or thermal activation may prevent us from reaching the top of the step where the emitted frequency is $8.236 \mathrm{GHz}$. (ii) Another possibility is the influence of the otherwise excluded admittance $Y_{S}$ of the bare Josephson element. We can estimate this admittance at $f_{1}$ by including $Y_{S}$ in Eq. (1). From the frequency difference at the top of the step, we find that the pair-current channel here acts as an inductance with $L_{S}=190 \mathrm{pH}$, which is $\sim 450$ times the minimum Josephson inductance, $L_{J}=(1 / 2 \pi)(h / 2 e)\left(1 / I_{c}\right)=$ $0.425 \mathrm{pH}$. This is in good agreement with our assumption of $Y_{S} \approx 0$. It is analogous to the theoretical prediction ${ }^{14}$ of $Y_{S}=0$ at the top of an rf-induced step. From $Y_{S}$, we can experimentally determine the effective junction plasma frequency on a self-induced mode step.

From recordings similar to Fig. 6, the quality factors $Q_{n}^{\text {expt }}, n=1,2$, and 3 were derived as the ratio of $f_{n}$ to the frequency difference $\Delta f_{n}$ measured at the half-power points. $\Delta f_{1}$ is $81.9 \mathrm{MHz}$, which yields $Q_{1}^{\text {expt }}=100.5$ and $G_{J}=0.093 \Omega^{-1}$. The theoretical value, $Q_{1}^{\text {theor }}$ is set equal to 100.5 in Table I, and with this fit the $Q$ values are calculated for the next modes. Despite that the experimental determination of $Q_{2}^{\text {expt }}$ and $Q_{3}^{\text {expt }}$ is rather uncertain ( $\pm 10 \%$ and $\pm 30 \%$, respectively), the agreement is far from convincing.

For the first two modes $G_{J}>G_{e}$ and here, the system losses are dominated by losses in the junction. The coupling parameter $z_{n}$ is much larger than the critical value 2.92 (single-resonance theory) for the first steps. At an external magnetic field of $100 \mu \mathrm{T}$, MS1 reaches its maximum size of $240 \mu \mathrm{A}$. The critical current at this field is $700 \mu \mathrm{A}$, giving $I_{1} / I_{c}=0.34$. Extrapolating from Fig. $12.6(m=1)$ in Ref. $1, I_{1} / I_{c}$ would be only about 0.05 for the fundamental lobe. Thus the step is much larger than predicted in the single-resonance theory.

The quasiparticle conductance at the voltage position of MS1 is measured by suppressing the supercurrent to zero. This yields $G_{J}=0.23 \Omega^{-1}$, which is a factor of 2 larger than the conductivity found in the $Q$ measurements. Even with $G_{J}=0.23 \Omega^{-1}, z_{1}$ is considerably larger than the critical value. The normal-state conductance of the junction just above the gap voltage is 0.62 $\Omega^{-1}$. Neither of these conductances fits the measurements. The effective high-frequency loss mechanisms of the junction need further consideration. We believe that 
the junction-resonator system is a fine candidate for use in this future work.

\section{CONCLUSION}

Very close coupling between a small Josephson tunnel junction and a multimode microstrip resonator has been utilized to study self-induced mode steps, MS $n$, and parametrically generated mode steps, PMS $k$. The coupling parameter can be tuned with an applied magnetic field. The calculated mode resonance frequencies are in good agreement with experiments, even though the admittance of the supercurrent path was neglected.

Compared with earlier experiments, we have used longer resonators, i.e., lower resonance frequencies, which in themselves favor a stronger coupling. Secondly, the junction capacitance loads the microstrip line and progressively shifts the resonant mode frequencies of the system. The shift, which depends on the ratio of the junction capacitance to the total capacitance of the external resonator, is kept relatively small in our experimental system with a small junction centered in a long low-impedance (wide and thin) stripline resonator.

We believe that the nearly equally spaced resonant mode frequencies, in combination with a large value of the coupling parameter, facilitate the observed subharmonic parametric excitation of the fundamental-mode resonance. The harmonic overlap also explains that both the PMS $k$ and MSn are considerably larger than predicted in the single-resonance theory. A large number of harmonics of the Josephson oscillations are nearly at resonance in the system, and hence their contributions to the nonlinear self-interactions cannot be neglected. Indirect evidence for strong coupling is that the PMS $k$ are split into separate bias-current intervals.

The mode resonance frequencies and the quality factors $Q_{n}$ are determined in situ by using the junction as a detector for externally applied microwave signals. The coupling parameter, which is deduced from the losses, is much larger than the critical value 2.92 found in the single-resonance theory. The junction losses dominate the system losses, but it is not possible to identify the measured losses with any conductance of the junction. From the difference between the fundamental-mode resonance frequency and the frequency of the Josephson ra- diation detected on MS1, it is likely that we may infer the effective inductance of the pair-current channel in a dynamic state. This is of interest in the study of nonlinear system dynamics.

Close coupling to junctions with dimensions longer than $\lambda_{J}$ can also be obtained by integration in resonant sections of transmission lines. In the case where the junction is small compared with the wavelength of the fundamental mode and with no appreciable harmonic overlap, this system will provide better control over the boundary condition for soliton motion, and permits experimental comparisons with recent simulations. ${ }^{21}$

The strongly coupled junction-microstrip system has potential applications as a narrow-band self-oscillating dc-biased microwave source. The available power is approximately given by the product of the dc voltage and bias current on the step. The generator impedance, however, is that of the resonator. What appears particularly attractive is that more of such systems can be coupled together via next-neighbor interactions in mutually phase-locked arrays. The usable power of a phase-locked array oscillator is proportional to the square of the number of systems, while the linewidth is inversely proportional to this number. Large zero-crossing microwaveinduced steps have been observed with weak power applied near the mode resonances. For Josephson voltage standards, this may be of practical importance, especially since the system opens the possibility of integrating the strongly coupled junction-resonator system as elements in a two-dimensional microstrip array antenna, thus avoiding present difficulties with circuit size when operating at lower frequencies as well as problems with distributing the power evenly to each junction.

\section{ACKNOWLEDGMENTS}

We thank R. Fromknecht, J. Niemeyer, and M.R. Samuelsen for stimulating discussions, R. Pöpel for help with the Mattis-Bardeen calculations and for use of his computer program for these calculations, and Anders Grunnet-Jepsen and Axel Morgenstjerne for assistance with some of the measurements. This work was partly funded by the Commission of the European Communities, the Danish Research Academy, and the Danish National Council of Metrology.
*Present address: Telecommunication Research Laboratory, DK-2970 Hørsholm, Denmark.

${ }^{1}$ K. K. Likharev, Dynamics of Josephson Junctions and Circuits (Gordon and Breach, New York, 1986), Chap. 12.

${ }^{2}$ G. Paterno, A. M. Cucolo, and G. Modestino, in Proceedings of the 17th International Conference on Low Temperature Physics LT-17, edited by U. Eckern, A. Schmid, W. Weber, and H. Wühl (North-Holland, Amsterdam, 1984) pp. 215-216.

${ }^{3}$ V. Yu. Kistenev, L. S. Kuzmin, A. G. Odintsov, E. A. Polunin, and L. Yu. Syromyatnikov, in SQUID-85, edited by H. D. Hahlbohm and H. Lübbig (W. de Gruyer, Berlin, 1985) pp. 113-118.

${ }^{4}$ A. D. Smith, B. J. Dalrymple, A. H. Silver, R. W. Simon, and J. F. Burch, IEEE Trans. Magn. MAG-23, 796 (1987).

${ }^{5}$ A. Raisanen, W. McGrath, P. Richards, and F. Lloyd, 
IEEE Trans. Microwave Theory Tech. MTT-33, 1495 (1985).

${ }^{6} \mathrm{~L}$. Kuzmin, H. K. Olsson, and T. Claeson, in SQUID-85 (Ref. 3), pp. 1017-1022.

${ }^{7}$ H. D. Jensen, A. Larsen, and J. Mygind (unpublished).

${ }^{8}$ J. E. Lukens, A. K. Jain, and K-L. Wan, in Superconducting Electronics, Vol. F59 of NATO Advanced Study Institute Series F, Computer and Systems Sciences, edited by $H$. Weinstock and M. Nisenoff (Springer-Verlag, Berlin, 1990), pp. 235-258.

${ }^{9}$ P. Hadley, M. R. Beasley, and K. Wiesenfeld, Phys. Rev. B 38, 8712 (1988).

${ }^{10}$ A. Larsen, H. D. Jensen, and J. Mygind (unpublished)

${ }^{11}$ H. D. Jensen, A. Larsen, and J. Mygind (unpublished).

${ }^{12}$ N. R. Werthamer and S. Shapiro, Phys. Rev. 164, 523
(1967).

${ }^{13}$ T. I. Smith, J. Appl. Phys. 45, 1875 (1974).

${ }^{14}$ R. L. Kautz, J. Appl. Phys. 52, 3528 (1981).

${ }^{15}$ N. F. Pedersen, O. H. Soerensen, B. Dueholm, and J. Mygind, J. Low Temp. Phys. 38, 1 (1980).

${ }^{16}$ D. C. Mattis and J. Bardeen, Phys. Rev. 111, 412 (1958).

${ }^{17}$ R. Pöpel, in Superconducting Quantum Electronics, edited by V. Kose (Springer, Berlin, 1989), pp. 44-78.

${ }^{18} \mathrm{R}$. Pöpel, IEEE Trans. Microwave Theory Tech. MTT-31, 600 (1983).

${ }^{19}$ R. Monaco, J. Appl. Phys. 68, 679 (1990).

${ }^{20} \mathrm{H}$. K. Olsson, Ph.D. thesis, Chalmers University of Technology, Göteborg, Sweden, 1988 (unpublished).

${ }^{21}$ N. Grønbech-Jensen, R. D. Parmentier, and N. F. Pedersen, Phys. Lett. A 142, 427 (1989). 